\title{
The onset of magnetic order in fcc-Fe films on $\mathrm{Cu}(100)$
}

\author{
S S A Razee ${ }^{1}$, J B Staunton ${ }^{1}$, L Szunyogh ${ }^{2}$, and B L Gyorffy ${ }^{3}$ \\ ${ }^{1}$ Department of Physics, University of Warwick, Coventry CV4 7AL, UK \\ ${ }^{2}$ Department of Theoretical Physics, Budapest University of Technology and Economics, \\ Budafoki út. 8, H-1521 Budapest, Hungary, and \\ Center for Computational Materials Science, Technical University of Vienna, Getreidemarkt \\ 9/158, A-1060 Vienna, Austria \\ ${ }^{3}$ H.H. Wills Physics Laboratory, University of Bristol, Tyndall Avenue, \\ Bristol BS8 1TL, UK
}

(November 14, 2018)

\begin{abstract}
On the basis of a first-principles electronic structure theory of finite temperature metallic magnetism in layered materials, we investigate the onset of magnetic order in thin (2-8 layers) fcc-Fe films on $\mathrm{Cu}(100)$ substrates. The nature of this ordering is altered when the systems are capped with copper. Indeed we find an oscillatory dependence of the Curie temperatures as a function of $\mathrm{Cu}$-cap thickness, in excellent agreement with experimental data. The thermally induced spin-fluctuations are treated within a mean-field disordered local moment (DLM) picture and give rise to layer-dependent 'local exchange splittings' in the electronic structure even in the paramagnetic phase. These features determine the magnetic intra- and interlayer interactions which are strongly influenced by the presence and extent of the $\mathrm{Cu}$ cap.

75.10.Lp, 75.40.Cx, 75.50.Bb, 75.70.Ak
\end{abstract}

Typeset using REVTEX 
The Disordered Local Moment (DLM) picture [1], when implemented by the KKR-CPA method [2], is a first-principles, that is to say material specific and yet parameter-free, mean field theory of metallic magnetism in the limit where good local moments form. It works well for bulk $\mathrm{Fe}$ and $\mathrm{Co}$ and their alloys but appears to be inadequate for $\mathrm{Ni}$ [3], [4]. Under these circumstances it is of fundamental interest to test its applicability by using it in different, but nevertheless similar, physical situations such as in a thin films whose thickness, $d$, can act as a tuneable, new thermodynamic variable. With this in mind we deployed the theory for $\mathrm{Fe}$ on and embedded in $\mathrm{Cu}$. In this letter we report some of our most significant findings.

For complexity in magnetic systems ultrathin $\mathrm{Fe}$ films on $\mathrm{Cu}(100)$ are unsurpassed [5]. How the films grow, their structure and morphology are profoundly intertwined with their magnetic properties and a satisfactory description of these systems has become a benchmark for theories of thin film magnetism. After much extensive experimental work on thermally deposited films it is now clear that below a critical thickness of 10-12 monolayers (ML) the films take on the fcc structure of the substrate while thicker films revert to the bcc structure of bulk iron. In the ultra thin regime when there are fewer than 4-5 ML a ferromagnetic (FM) phase is observed whilst the thicker films of 6-11 ML seem to be antiferromagnetic (AF) with a net moment across the film. Adding covering layers of $C u$ to the films has a marked effect upon their magnetic properties. A single $C u$ monolayer suppresses the magnetic ordering temperatures $T_{c}$ whilst the $T_{c}$ 's of $2 \mathrm{ML} \mathrm{Cu}$-capped films are partly restored. In fact $T_{c}$ oscillates weakly as further $\mathrm{Cu}$ layers are added [6].

The experimental data on the uncapped films has been interpreted [7] in terms of models in which the top two surface iron layers are coupled together ferromagnetically in an otherwise AF Fe film. This picture has been prompted by a series of ab-initio $T=0 \mathrm{~K}$ electronic structure total energy calculations [8 10]. Pajda et al. [1] have calculated effective exchange interactions in a $C u$-capped $F e$ monolayer on $C u(100)$ and obtained an oscillatory variation of $T_{c}$ with cap thickness. Here we describe the full DLM picture of both electronic and magnetic structure pertinent to the paramagnetic states of $F e$-films on $C u(100)$ of up to 8ML thickness at finite temperatures and investigate both the growth of 
magnetic correlations and onset of magnetic order. Moreover we are able to extract intraand interlayer 'exchange' interactions.

The DLM picture is based on assuming a separation between fast and slow degrees of freedom in the interacting many-electron system. For times, $\tau$, long in comparison with an electronic hopping times, $\hbar / W\left(\approx 10^{-15}\right.$ seconds), where $W$ represents a relevant bandwidth, but short when compared with typical spin fluctuation times, the spin orientations of the electrons leaving an atomic site are sufficiently correlated with those arriving that the magnetisation integrated over a unit cell and averaged over $\tau$ is non-zero. These are the 'local moments' which can change their orientations, described by a set of unit vectors $\left\{\hat{e}_{i}\right\}$ labelled by the site index $i$, on a time scale longer than $\tau$ while their magnitudes fluctuate rapidly on the time scale $\tau$. This timescale demarcation has been invoked in much recent work 12 15 on bulk systems but has not been examined and tested in thin film systems. This is the issue we address in this letter.

For an ab-initio implementation of this picture, standard Spin Density Functional theory for studying electrons in spin-polarised metals is adapted to describe the states of the system for each orientational configuration $\left\{\hat{e}_{i}\right\}$. At the heart of the theory is the generalised electronic Grand Potential $\Omega\left\{\hat{e}_{i}\right\}$ of the system so constrained. A long time average can be replaced by an ensemble average with respect to the Gibbsian measure $P\left\{\hat{e}_{i}\right\}=Z^{-1} \exp -\beta \Omega\left\{\hat{e}_{i}\right\}$, where the partition function $Z=\prod_{i} \int d \hat{e}_{i} \exp -\beta \Omega\left\{\hat{e}_{i}\right\}$. The thermodynamic free energy, which accounts for the entropy associated with the orientational fluctuations as well as creation of electron-hole pairs, is given by $F=-k_{B} T \log Z$. Evidently $\Omega\left\{\hat{e}_{i}\right\}$ plays the role of a classical 'spin' (local moment) Hamiltonian, albeit a highly complicated one. By choosing a suitable reference single-site 'spin' Hamiltonian $\Omega_{0}\left\{\hat{e}_{i}\right\}=\sum_{i} \omega_{i}\left(\hat{e}_{i}\right)$ and using the Feynman-Peierls' inequality [16] a mean field theory is constructed [2, [4]. This is a 'first principles' formulation of the Disordered Local Moment (DLM) picture which can be implemented by an adaptation of SCF-KKR-CPA method ideally suited for calculating the partial averages $\omega_{i}\left(\hat{e}_{i}\right)$. The approach can be further improved via the construct of a generalised Onsager cavity field [四]. 
When the above procedure was used to study bulk magnetic metals, it was found that the local electronic structure can possess a 'local exchange' splitting even in the paramagnetic state [2]. This means that an electron spin-polarised parallel to a local moment will have a different density of states to that polarised anti-parallel. When all orientations of the moments are averaged over in the paramagnetic state the electronic structure is inevitably unpolarised but consequences from the presence of local moments can still be identified. (Moreover the magnitude of this splitting is expected to vary sharply as a function of wavevector and energy. At some points, if the 'bands' are flat in a region of wave-vector and energy space, the local exchange splitting will be roughly of the same size as the rigid exchange splitting of the majority and minority-spin bands of the ferromagnetic state whereas at other points, where the 'bands' have greater dispersion, the splitting will vanish entirely.) This local exchange-splitting is the cause of the establishment of a local moment. Photoemission [17] and inverse photoemission (IPES) experiments [18] have found these qualitative features in bcc $F e$. In our generalisation of the DLM theory to layered systems we find that the layer dependence of similar features in the electronic structure of paramagnetic $F e$ grown on a $C u(100)$ substrate drives the onset of magnetic order and the form of the magnetic interactions between $F e$ layers.

To probe the content of the theory consider the response of the paramagnetic DLM state to the application of a small, external spin-only magnetic field, $\left\{\mathbf{h}_{\mathbf{i}}\right\}$, varying from site to site, layer to layer in both orientation and magnitude. The induced magnetisation is predominantly caused by the local moments changing their orientations to align with the field causing a change in the single-site probabilities, $\delta P_{i}\left(\mathbf{e}_{i}\right)$. In short the site by site paramagnetic spin susceptibility [3] is the solution of the matrix equation,

$$
\chi^{i j}=\frac{\beta}{3} \mu_{i}^{2} \delta_{i j}+\frac{\beta}{3} \sum_{k} S_{i k}^{(2)} \chi^{k j}
$$

where $S_{i k}^{(2)}$ is the corresponding direct correlation function and $\mu_{i}$ is the magnitude of the local magnetic moment on the $i$-th site. Expressions for $S_{i j}^{(2)}$ involving the electronic structure of the paramagnetic state and techniques for calculating them, using the KKR-CPA, for 
bulk systems have been given elsewhere [3]. For layered systems with two-dimensional translational symmetry the magnitudes of the 'local moments', $\left\{\mu_{i}\right\}$, assume one value $\mu_{P}$ per site in a given layer labelled $P$ but vary from layer to layer. Taking a 2D lattice Fourier transform over sites within each layer equation (11) can be rewritten as,

$$
\begin{aligned}
\chi^{P Q}\left(\mathbf{q}_{\|}\right) & =\sum_{i j} \chi^{P i Q j} \exp \left[-i \mathbf{q}_{\|} \cdot\left(\mathbf{R}_{i}-\mathbf{R}_{j}\right)\right] \\
& =\frac{\beta}{3} \mu_{P}^{2} \delta_{P Q}+\frac{\beta}{3} \sum_{S} S_{P S}^{(2)}\left(\mathbf{q}_{\|}\right) \chi^{S Q}\left(\mathbf{q}_{\|}\right)
\end{aligned}
$$

where, $\mathbf{q}_{\|}$is a wave-vector in the $2 \mathrm{D}$ layer Brilliouin Zone. Once the $S_{P S}^{(2)}\left(\mathbf{q}_{\|}\right)$'s have been obtained from the SCF-KKR-CPA calculations and loaded into a $n \times n$ matrix, $S^{(2)}\left(\mathbf{q}_{\|}\right)$ (where $n$ is the number of layers in the film), this set of equations (2) is solved by a simple matrix inversion i.e. $\chi^{P Q}\left(\mathbf{q}_{\|}\right)=\left[3 k_{B} T I-S^{(2)}\left(\mathbf{q}_{\|}\right)\right]_{P Q}^{-1} \mu_{Q}^{2}(I$ is a unit matrix $)$. For films in which the intralayer exchange coupling is ferromagnetic, as for the $F e$ films on $C u$, the Curie temperature $T_{c}$ is specified by the condition $\left\|3 k_{B} T_{C} I-S^{(2)}\left(\mathbf{q}_{\|}=0\right)\right\|=0$ or in terms of the largest eigenvalue of $S^{(2)}\left(\mathbf{q}_{\|}=0\right)$. Full technical details on how $S^{(2)}\left(\mathbf{q}_{\|}\right)$is calculated for layered systems will be provided elsewhere.

We use the spin-polarised screened KKR method [19] for layered systems adapted for the DLM picture. The lattice constants of the layers are taken to be the same as that of the substrate (6.83 a.u.) so that the effects of lattice strain on the electronic structure are neglected. For each $n$, the electronic structure of the DLM state is calculated self-consistently using $78 k_{\|}$points in the irreducible part of the surface Brillouin zone. A buffer of three layers of the substrate as well as a buffer of at least three layers of vacuum is calculated self-consistently along with the potentials on each layer. The self-consistent layer-resolved potentials are then used to calculate the magnetic moments and the effective 'Weiss' field on each layer as described above. By introducing a small change in the average magnetisation on one particular layer and calculating the effective 'Weiss' field on each layer we determine $S_{P Q}^{(2)}\left(\mathbf{q}_{\|}=0\right)$, and hence the static paramagnetic spin susceptibility, $\chi^{P Q}\left(\mathbf{q}_{\|}=0\right)$ together with the Curie temperatures $T_{c}$ 's for various thicknesses of both the $\mathrm{Fe}$ film and the $\mathrm{Cu}$ cap. 
Table [- shows our results for the layer-dependent 'local' magnetic moments in uncapped $\mathrm{Fe}_{n} / \mathrm{Cu}(100)$ films together with the dependence of the Curie temperatures on Fe-film thickness. The moments on the topmost layer are always the largest at around $2.5 \mu_{B}$ followed by the moments on the Fe layer closest to the substrate next at roughly $2.2 \mu_{B}$. In the interior of the film the moments are reduced to $1.7 \mu_{B}$ close to what was found for fcc-Fe for this lattice spacing [20]. The magnetic ordering temperatures reduce monotonically to a constant value of $485 \mathrm{~K}$ for $6 \mathrm{MLs}$ onwards. Table $\llbracket$ shows the effective 'exchange' interactions, that is to say the direct correlation function, $S_{P Q}^{(2)}$ which lead to these transition temperatures. Examples of a 7ML and a 3ML Fe layer systems are shown to bring out the dominant features. The intralayer couplings are ferromagnetic throughout. The layer at the top of film and the layer nearest the $\mathrm{Cu}$ substrate have the largest values owing to their reduced effective coordination. It is the interlayer couplings that show an important and interesting trend. The top two layers are strongly ferromagnetically (FM) coupled (positive values) whereas the coupling to nearest neighbor layers within the films is anti-ferromagnetic (AF). There is also significant coupling to subsequent layers which alternates between FM and AF.

Adding a copper cap to these films alters these results profoundly. Figure 11 highlights this for 3 and 7 monolayer $\mathrm{Fe} / \mathrm{Cu}(100)$ films and shows the magnetic ordering temperature $T_{c}$ to have an oscillatory dependence on cap thickness. A single $\mathrm{Cu}$ monolayer suppresses $T_{c}$ by some $50 \mathrm{~K}$ with the deficit being restored by a second layer. A third layer reduces $T_{c}$ once again and further layers cause minor oscillations about this lower value. This behavior is in excellent agreement with experimental data on the same systems by Vollmer et al. [6] (see their figure 6). In figure 2 the dominant changes to intra- and interlayer magnetic interactions are shown. These occur in the top two Fe layers nearest the cap. A single $\mathrm{Cu}$ ML cap switches the coupling between these layers from FM to AF whilst further Cu layers strengthen the coupling within the topmost Fe layer.

Calculations for bulk fcc-Fe have shown how the tendency to magnetic order changes as the lattice spacing is varied [21,20]. On an expanded lattice, a ferromagnetically ordered state is stable at low temperatures and the coupling between 'local moments' in the param- 
agnetic DLM state mirrors this aspect. As the lattice is contracted the magnetic ordering tendencies become anti-ferromagnetic. Capping fcc Fe films with $\mathrm{Cu}$ causes a similar effect. Figure 3 shows that the electronic density of states (DOS) of an uncapped film is 'exchange split' over a large energy range. These states are nearly fully occupied and FM coupling between neighboring sites results. The reduced coordination of sites within this layer produce an effect akin to that of increasing the lattice spacing of bulk fcc Fe. There is hybridisation of these Fe states with quantum well states in the $\mathrm{Cu}$ cap formed between the vacuum and the Fe film. This causes the exchange splitting to collapse in the region of this hybridisation just as in fcc Fe for smaller lattice spacings. The remaining exchange-split states are roughly half-filled which leads an AF coupling along the (100) direction as in bulk fcc Fe. Figure 3 shows these effects in the DOS in the top layer of a $\mathrm{Fe}_{7} / \mathrm{Cu}(100)$ system both uncapped and capped by a single $\mathrm{Cu} \mathrm{ML}$. The narrow band of states in the $\mathrm{Cu}$ layer are also shown.

In conclusion we note that the dramatic variations of interaction between local moments at different distances from the various surfaces and interfaces are quintessentially itinerant effects. As the much studied variation of the local moments near such planar defects show, they are due to the motion of the electrons not limited to an atom but confined by the geometry of the sample. In this respect they are manifestations of the same physics as is at work in the oscillatory magnetic coupling in metallic multilayers 22]. Consequently, the detailed quantitative account of the complex experimental data by our calculations can be taken as significant new evidence that the first-principles (KKR-CPA) implementation of the DLM picture correctly captures the essential physics of magnetic order, due to mobile electrons, in the limit where local moments form.

Acknowledgments: This work is a result of a collaboration under the TMR-Network (contract no. ERBFMRXCT 96-0089) on "Ab initio calculations of magnetic properties of surfaces, interfaces, and multilayers" and also the RTN 'Computational Magnetoelectronics' network (contract number HPRN-CT-2000-00143) and is supported by the Engineering and Physical Sciences Research Council (UK) and the Hungarian National Science Foundation (contracts OTKA T030240 and T029813). 


\section{REFERENCES}

[1] J.Hubbard, Phys. Rev.B 20, 4584, (1979); H.Hasegawa, J. Phys. Soc. Japan 461504 , (1979); D.M.Edwards, J. Phys.F 12 1789, (1982).

[2] B.L.Gyorffy et al. J. Phys. F 15 1337,(1985); J.B.Staunton et al., J. Phys.F 15, 1387, (1985).

[3] J.B.Staunton et al., J. Phys.F 16 1761, (1986)

[4] J.B.Staunton and B.L.Gyorffy, 1992, Phys.Rev.Lett. 69, 371.

[5] M.Straub et al., Phys.Rev.Lett. 77, 743, (1996).

[6] R.Vollmer et al., Phys.Rev.B 61, 1303 (2000).

[7] R.E.Camley and Dongqi Li, Phys.Rev.Lett. 84, 4709, (2000).

[8] C.L.Fu and A.J.Freeman, Phys.Rev.B 35, 925, (1987); G.W.Fernando and B.R.Cooper, Phys.Rev.B 38, 3016, (1988); T.Kraft et al., Phys.Rev.B 49, 11511, (1994).

[9] R. Lorenz and J. Hafner, Phys. Rev. B 54, 15937 (1996); L. Szunyogh et al., Phys. Rev. B 55, $14392(1997)$

[10] T.Asada and S.Blugel, Phys.Rev.Lett. 79, 507, (1997).

[11] M.Pajda et al., Phys.Rev.Lett. 85, 5424, (2000).

[12] M.Uhl and J.Kubler, Phys.Rev.Lett. 77, 334, (1996).

[13] A.I.Liechtenstein et al., J.Mag.Magn.Mat. 67, 65, (1987).

[14] M. van Schilfgaarde and V.P.Antropov, J.Appl.Phys. 85, 4827, (1999).

[15] M.Pajda et al., cond-mat/0007441.

[16] R.P.Feynman, Phys.Rev. 97, 660, (1955).

[17] E.Kisker et al., Phys.Rev.Lett. 52, 2285, (1984). 
[18] J.Kirschner et al., Phys.Rev.Lett. 53, 612, (1984).

[19] L.Szunyogh et al., Phys.Rev.B 51, 9552, (1995); R.Zeller et al., Phys.Rev.B 52, 8807, (1995); L.Szunyogh et al., Phys.Rev.B 492721 (1994).

[20] F.J.Pinski et al., Phys.Rev.Lett. 56, 56, 2096, (1986).

[21] C.S.Wang et al., Phys.Rev.Lett. 54, 1852, (1985); V.L.Moruzzi and P.M.Marcus, 'Energy band theory of metallic magnetism in the elements',Handbook of Magnetic Materials,7, eds: K.H.J.Buschow (Amsterdam: North Holland), 97, (1993).

[22] P.Bruno and C.Chappert, Phys.Rev.Lett. 67, 1602, (1991); E.Bruno and B.L.Gyorffy, J.Phys. Condens. Matter 5, 2109, (1993). 


\section{TABLES}

TABLE I. 'Local' magnetic moments in $\mu_{B}$ on different layers and the Curie temperatures of uncapped $\mathrm{Fe}_{n} / \mathrm{Cu}(100)$ systems. The Fe Layer $L_{1}$ is adjacent to the substrate and layer $L_{n}$ is the top layer.

\begin{tabular}{|c|c|c|c|c|c|c|c|c|c|}
\hline & \multicolumn{8}{|c|}{ 'Local' moments $\left(\mu_{B}\right)$} & \multirow[t]{2}{*}{$T_{c}(\mathrm{~K})$} \\
\hline $\mathrm{n}$ & $L_{1}$ & $L_{2}$ & $L_{3}$ & $L_{4}$ & $L_{5}$ & $L_{6}$ & $L_{7}$ & $L_{8}$ & \\
\hline 2 & 2.24 & 2.48 & & & & & & & 681 \\
\hline 3 & 2.21 & 1.70 & 2.53 & & & & & & 532 \\
\hline 4 & 2.21 & 1.67 & 1.74 & 2.53 & & & & & 495 \\
\hline 5 & 2.21 & 1.67 & 1.71 & 1.74 & 2.53 & & & & 492 \\
\hline 6 & 2.21 & 1.67 & 1.71 & 1.71 & 1.74 & 2.53 & & & 486 \\
\hline 7 & 2.21 & 1.67 & 1.71 & 1.72 & 1.71 & 1.74 & 2.53 & & 485 \\
\hline 8 & 2.21 & 1.67 & 1.71 & 1.72 & 1.71 & 1.71 & 1.74 & 2.53 & 485 \\
\hline
\end{tabular}

TABLE II. Intra- and inter-layer effective 'exchange' interactions in meV between layers in an uncapped $\mathrm{Fe}_{7} / \mathrm{Cu}(100)$ system with the values for $\mathrm{Fe}_{3} / \mathrm{Cu}(100)$ shown for comparison in brackets. The Fe Layer $L_{1}$ is adjacent to the substrate and layer $L_{n}$ is the top layer.

\begin{tabular}{|c|c|cccc|cc|}
\hline \hline $\mathrm{n}$ & $L_{1}$ & $L_{2}$ & $L_{3}$ & $L_{4}$ & $L_{5}$ & $L_{6}$ & $L_{7}$ \\
\hline 1 & $108.5(108.4)$ & -7.5 & 8.5 & -2.1 & -0.8 & $-0.7(-10.9)$ & $-1.0(25.1)$ \\
\hline 2 & -7.5 & 19.6 & -7.5 & 3.2 & -2.2 & -0.6 & -0.7 \\
3 & 8.5 & -7.5 & 17.5 & -7.2 & 2.1 & -1.7 & -0.7 \\
4 & -2.1 & 3.2 & -7.2 & 18.8 & -8.1 & 2.1 & -2.1 \\
5 & -0.8 & -2.2 & 2.1 & -8.1 & 19.9 & -10.0 & 9.8 \\
\hline 6 & $-0.7(-10.9)$ & -0.6 & -1.7 & 2.1 & -10.0 & $33.2(29.9)$ & $50.0(53.8)$ \\
7 & $-1.0(25.1)$ & -0.7 & -0.7 & -2.1 & 9.8 & $50.0(53.8)$ & $97.7(96.7)$ \\
\hline \hline
\end{tabular}




\section{FIGURES}

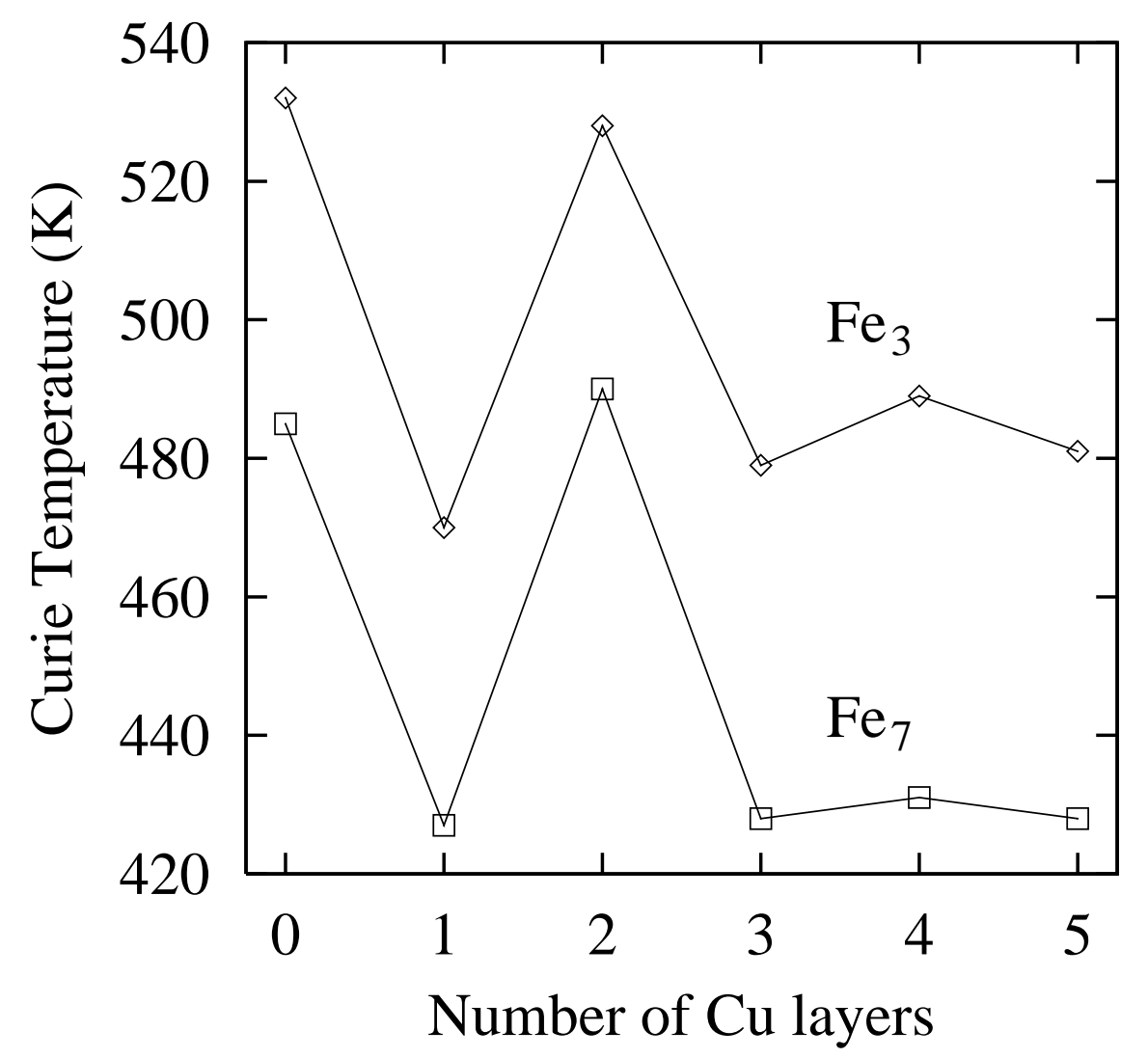

FIG. 1. The magnetic ordering temperatures for 3 and 7 layers of $F e$ on $C u(100)$ as a function of number of copper capping layers. 


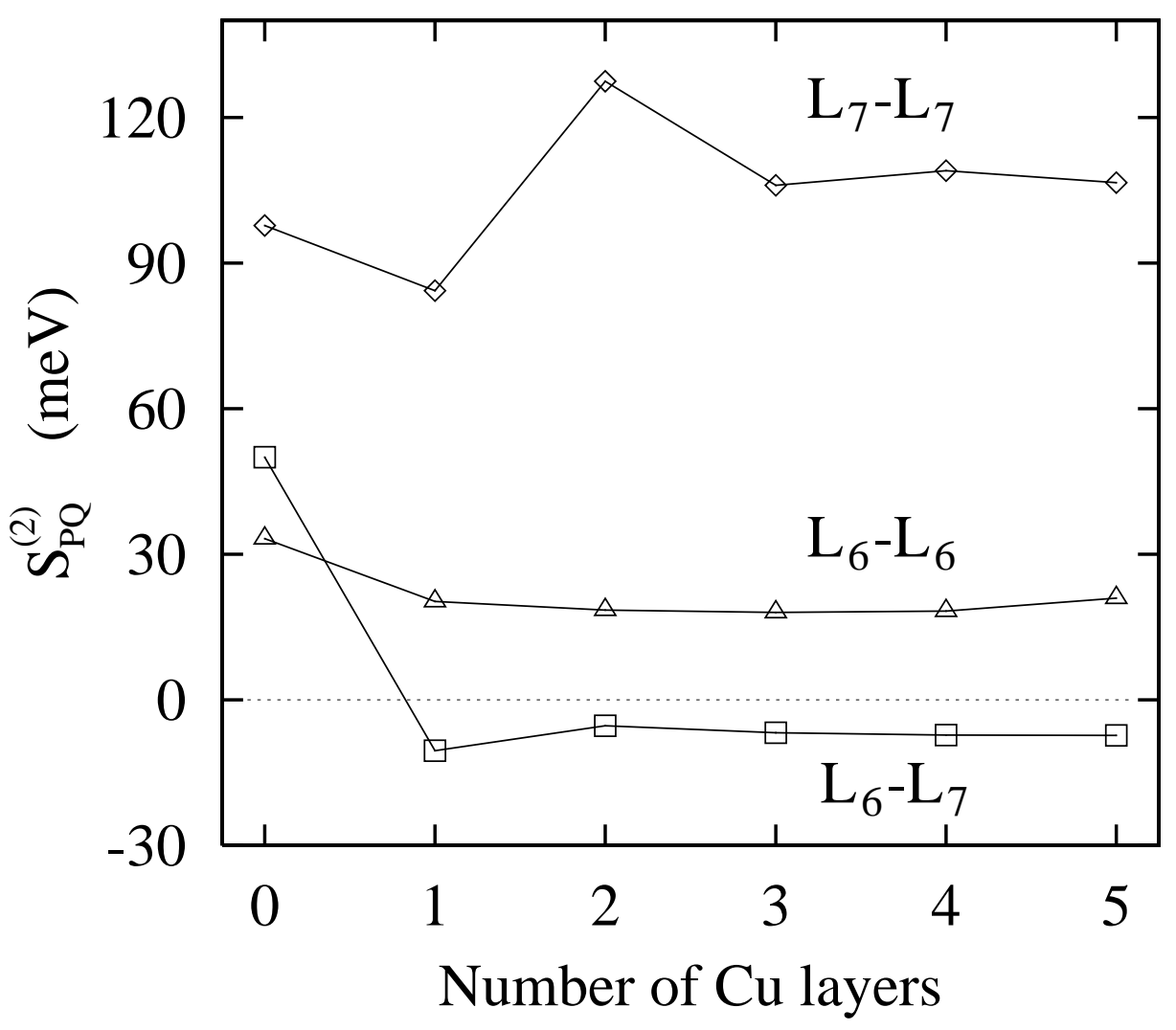

FIG. 2. The magnetic interactions in and between the $F e$ layers nearest the surface or copper cap in a system of $7 \mathrm{Fe}$ layers on $C u(100)$. The values are given in $\mathrm{meV}$ and ' $L_{7}-L_{7}$ ' denotes the interactions within the top $F e$ layer (nearest the cap), ' $L_{6}-L_{6}$ ' for the second-most top layer and ' $L_{6}-L_{7}$ ' the coupling between these two iron layers. 


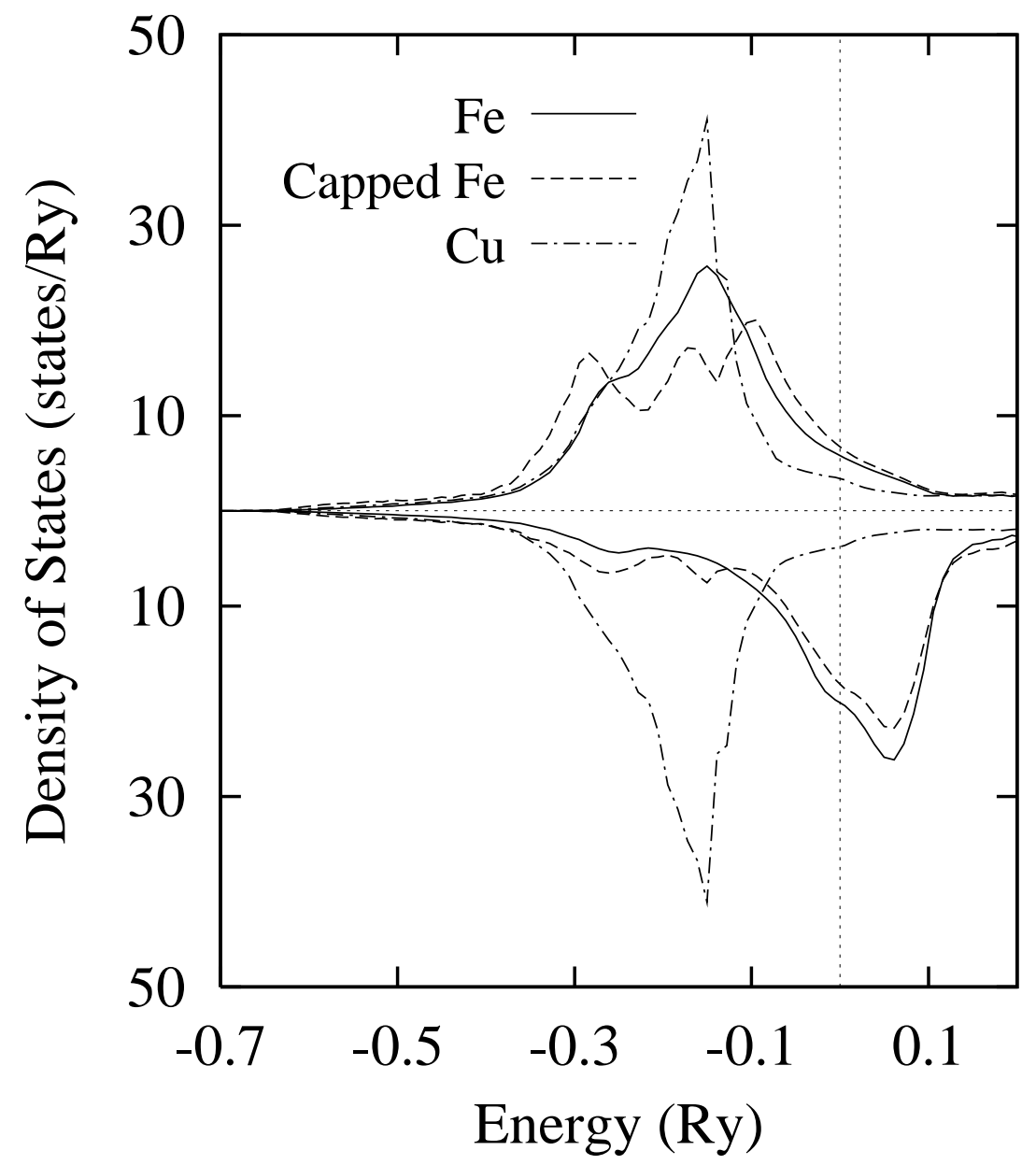

FIG. 3. The density of states (DOS) in the topmost Fe layer of a $\mathrm{Fe}_{7} \mathrm{Cu}(100)$ system. The full line shows the uncapped system whilst the dashed line shows the DOS when a single Cu monolayer caps the system. The dotted line shows the DOS for this $\mathrm{Cu}$ layer. The upper half of the figure shows the DOS for an electron spin-polarised parallel to the local moment on a site whereas in the lower half the DOS of an electron polarised anti-parallel is shown. 